\title{
Experiencia pedagógica para la enseñanza de la biomecánica mediada por software en escuelas de
}

ingeniería biomédica

Juana Yadira Martín-Perico ${ }^{1}$

Universidad Santo Tomás

juanamartin@usantotomas.edu.co

Bernardo Garibello-Suan ${ }^{2}$

Universidad Santo Tomás

bernardogaribello@usantotomas.edu.co

DOI: https://doi.org/10.21158/2357514x.v7.n2.2019.2465

Cómo citar este artículo: Martín-Perico, J. Y.; Garibello-Suan, B. (2019). Experiencia pedagógica para la enseñanza de la biomecánica mediada por software en escuelas de ingeniería biomédica. Revista Virtu@Imente, 7(2), 9-26.

DOI: https://doi.org/10.21158/2357514x.v7.n2.2019.2465

Fecha de recepción: 06 de mayo 2019

Fecha de aprobación: 16 de octubre de 2019
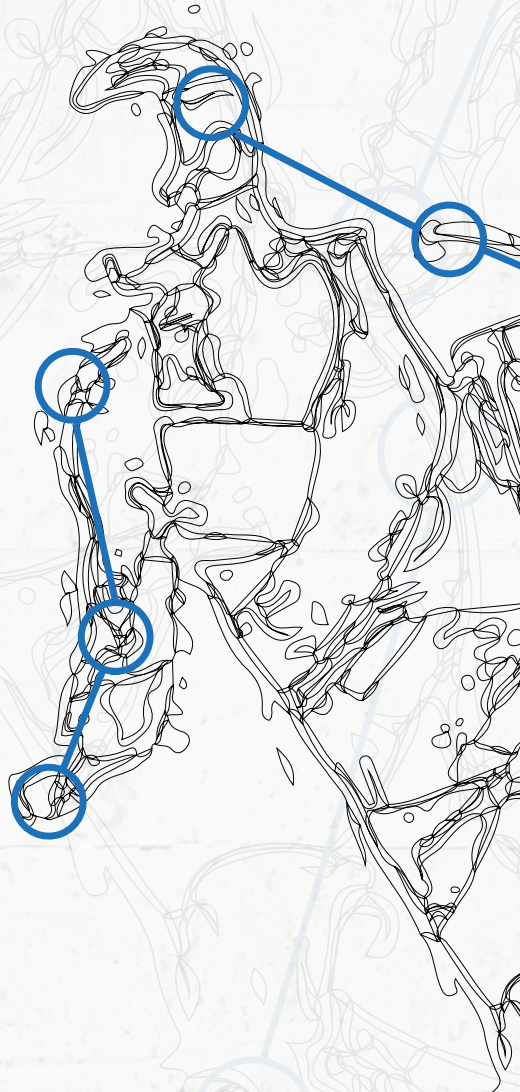

${ }_{1}^{1}$ Doctora en educación - Universidad Santo Tomás. Especialista en instrumentación electrónica - Universidad Santo Tomás. Ingeniera Biomédica - Universidad Manuela Beltrán. ORCID: https://orcid.org/0000-0001-5416-1393

${ }^{2}$ Magister en modelado y simulación - Universidad Jorge Tadeo Lozano-Universidad Central. Ingeniero electrónico - Universidad Nacional de Colombia. Licenciado en Matemática - Universidad Pedagógica. ORCID: https://orcid.org/0000-0003-0366-4710 
El presente documento contiene la descripción de una experiencia de aula desarrollada en una escuela de ingeniería biomédica de Bogotá, cuya estrategia fue emplear el software de análisis de movimiento como mediación pedagógica a fin de articular al estudiante en el marco de la sociedad del conocimiento y sus recursos, lo que lo llevó a contrastar los conceptos de biomecánica con lo observado en la tecnología y así estar en capacidad de caracterizar el cuerpo humano en aplicaciones de clínica, deporte e investigación. El ejercicio didáctico propendió a minimizar la reprobación académica en el área; incrementó la capacidad descriptiva, de síntesis y de argumentación del estudiante; permitió que los discentes propusieran ideas para sus proyectos de grado y brindó la oportunidad a los docentes de articular lo trabajado en el aula con la práctica de laboratorio a fin de trabajar proyectos transdisciplinares. La contribución del software en el análisis de movimiento en situaciones biomecánicas patológicas es una oportunidad para que el estudiante comprenda las condiciones de adaptación del cuerpo humano, lo cual conduce al desarrollo de habilidades especiales que son de gran interés para proponer diseños de ayudas funcionales y la creación de dispositivos biomédicos para contribuir de esta manera en la mejoría de la calidad de vida de las personas a través del incremento de los desarrollos de ingeniería biomédica en campos como las ayudas funcionales, los dispositivos de rehabilitación y la protección industrial.

Palabras clave: experiencia de aula; mediación pedagógica; enseñanza de la biomecánica; didáctica de la biomecánica; uso de la tecnología para la enseñanza; software educativo. 


\section{Pedagogical experience for teaching software-mediated biomechanics in schools of biomedical engineering}

\section{ABSTRACT}

This document contains the description of a classroom experience developed in a biomedical engineering school in Bogotá, whose strategy was to use the movement analysis software as a pedagogical mediation in order to articulate the student in the framework of the society of knowledge and its resources, which led to contrast the concepts of biomechanics with what was observed in technology, and thus be able to characterize the human body in clinical, sports, and research applications. The didactic exercise aimed at minimizing the academic failure in the area; it increased the capabilities of description, synthesis, and argumentation of students; it allowed them to propose ideas for their degree projects and provided the opportunity for teachers to articulate the work that had been done in the classroom with the laboratory practice, so as to work on transdisciplinary projects. The contribution of the software in the analysis of movement in pathological biomechanical situations is an opportunity for the student to understand the conditions of adaptation of the human body, which leads to the development of special skills that are of great interest for proposing functional aid designs and for the creation of biomedical devices, in order to contribute to the improvement of the quality of life of people through the increase of biomedical engineering development in fields such as functional aids, rehabilitation devices, and industrial protection.

Keywords: classroom experience; pedagogical mediation; biomechanics education; biomechanics didactics; use of technology for education; educational software. 


\title{
Experiência pedagógica no ensino de biomecânica mediada por software em escolas de engenharia biomédica
}

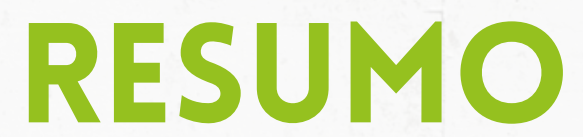

\begin{abstract}
Este documento contém a descrição de uma experiência realizada na sala de aula de uma escola de engenharia biomédica em Bogotá, cuja estratégia era usar o software de análises de movimento como mediação pedagógica, a fim de insertar o aluno dentro da estrutura da sociedade do conhecimento e seus recursos, o que o levou a contrastar os conceitos de biomecânica com os observados na tecnologia e, assim, ser capaz de caracterizar o corpo humano em aplicações clínicas, esportivas e de pesquisa. O exercício didático tendia a minimizar a desaprovação acadêmica na área; aumentou a capacidade descritiva, de síntese e argumentação do aluno; isso permitiu que os alunos apresentassem ideias para seus projetos de graduação e proporcionou aos professores a oportunidade de articular o que foi desenvolvido em sala de aula com a prática de laboratório para trabalhar em projetos transdisciplinares. A contribuição do software na análise do movimento em situações biomecânicas patológicas é uma oportunidade para o aluno entender as condições de adaptação do corpo humano, o que leva ao desenvolvimento de habilidades especiais de grande interesse para propor projetos de auxílio funcional e criação de dispositivos biomédicos para contribuir dessa maneira com a melhoria da qualidade de vida das pessoas, aumentando os desenvolvimentos de engenharia biomédica em áreas como auxiliares funcionais, dispositivos de reabilitação e proteção industrial.
\end{abstract}

Palavras-chave: experiência em sala de aula; mediação pedagógica; educação em biomecânica; ensino de biomecânica; uso de tecnologia para ensino; software educacional. 


\section{Expérience pédagogique de l'enseignement de la biomécanique assistée par logiciel numérique dans les écoles d'ingénierie biomédicale}

\section{RÉSUMÉ}

Ce document décrit une expérience de classe réalisée dans une école d'ingénierie biomédicale de Bogotá via l'utilisation d'un logiciel d'analyse de mouvement dans le cadre d'une séance pédagogique de mise à niveau des connaissances des étudiants dans ce domaine. Les concepts de biomécanique et de technologie n'y sont pas opposés et les étudiants peuvent ainsi mieux comprendre le corps humain via des applications cliniques, sportives et $d^{\prime}$ investigations. Cet exercice didactique a permis de minimiser la désapprobation académique; d'augmenter la capacité de description, de synthèse et d'argumentation des étudiants, leur permettant de trouver des problèmatiques pour leurs projets de diplôme en donnant l'occasion aux enseignants d'articuler le travail de classe avec la pratique de laboratoire afin d'élaborer des projets transdisciplinaires.

L'apport du logiciel dans l'analyse des mouvements biomécanico-pathologiques donne l'opportunité aux étudiant de comprendre les conditions d'adaptation du corps humain, leur permet d'acquèrir des compétences particulières recherchées pour la conception de supports fonctionnels ou pour la création de dispositifs biomédicaux qui contribuent à l'amélioration de la qualité de vie des patients et au développement de l'ingénierie biomédicale dans des domaines tels que les aides fonctionnelles, les dispositifs de rééducation et la protection industrielle.

Mots-clés: expérience de classe; médiation pédagogique; éducation à la biomécanique; enseignement de la biomécanique; utilisation de la technologie pour l'enseignement; logiciel éducatif. 


\section{Introducción}

En las escuelas de ingeniería biomédica el área de biomecánica constituye una de las líneas de investigación y desarrollo disciplinar más importante para los futuros profesionales. Esto en razón a que su estudio y comprensión dota al estudiante de los elementos conceptuales necesarios para entender los efectos de las fuerzas externas e internas que interactúan sobre el cuerpo humano y sus posibles efectos (Poveda-Polo, 2011). Sin embargo, en la práctica académica una gran cantidad de conceptos y relaciones -estudiadas en este curso- suelen ser de difícil comprensión para los estudiosos, pues requieren de la observación minuciosa de situaciones y fenómenos en dos y tres dimensiones, además de la comprensión, el análisis de algoritmos matemáticos abstractos y relaciones físicas de alta complejidad, así como del manejo de conceptos integrados de biología, anatomía, fisiología y química (Aguilar y Gutiérrez, 2000).

Lo anterior evidencia la necesidad de establecer escenarios de aprendizaje que posibiliten la comprensión y la aplicación de las biociencias. Para esto se emplea una estrategia didáctica que contemple escenarios académicos provistos con recursos tecnológicos de captura, visualización y procesamiento de datos (Frutos y Palao, 2012) con los que el estudiante interactúe, analice y pueda proponer soluciones a los problemas de índole estructural y funcional que -es posible- encuentre en su práctica, a fin de construir su propio conocimiento (Bergós y Cabrera, 2014). Con la finalidad de llevar a cabo la experiencia didáctica se medió esta a través del empleó del software de análisis de movimiento, como una estrategia de aprendizaje orientada a fomentar la comprensión temporo-espacial, la identificación de conceptos de biociencias y ciencias exactas y la capacidad analítica de los 95 estudiantes inscritos en el curso de Biomecánica y Mecanismos, en los periodos 2018I, 2018-II y 2019-I, en un programa de ingeniería biomédica de Bogotá.

La estrategia metodológica trazada para implementar la didáctica consistió en el desarrollo de un proyecto de aula en el que los estudiantes, guiados por el profesor titular, se organizaron por equipos de trabajo con el propósito de desarrollar guías de laboratorio, previamente estructuradas por los docentes, con las que les fue posible establecer una relación de lo visto en clase con su práctica, además de analizar por medio del software el cuerpo humano regular y patológico. Así, establecieron las diferencias significativas, lo que los llevó a proponer posibles soluciones a las problemáticas identificadas a través de rutinas, ayudas funcionales o prototipos. Lo anterior expone escenarios en los que los profesores universitarios pueden alimentar sus prácticas de aula, enriquecer sus procesos didácticos, mejorar la atención, incrementar el interés de los estudiantes en las biociencias $y$, finalmente, en los que los docentes tienen la posibilidad de dar a conocer nuevas estrategias de enseñanza en el área de la biomecánica, ya que la información encontrada suele ser escasa. 


\section{Tensiones en la enseñanza-aprendizaje de la biomecánica en una escuela de ingeniería biomédica}

La biomecánica, como disciplina, se vale de diversas áreas del conocimiento para estudiar los sistemas vivos a través de los principios de la mecánica. Esto con el fin de identificar los efectos que tienen sobre ellos la energía y las fuerzas, así como establecer modelos representativos (Donskoi y Zatsiorski, 1988; Dufour y Pillu, 2018). Estos se analizan de manera amplia a través de investigaciones que llevan a la propuesta de diversas aplicaciones y han de contribuir a mejorar la salud y la calidad de vida de las personas, tales como los modelos de los sistemas del cuerpo humano, el sistema músculo-esquelético, el respiratorio, el cardiovascular y el cardiopulmonar (véase una síntesis de esto en la figura 1).

Figura 1. La biomecánica y su relación con otras disciplinas

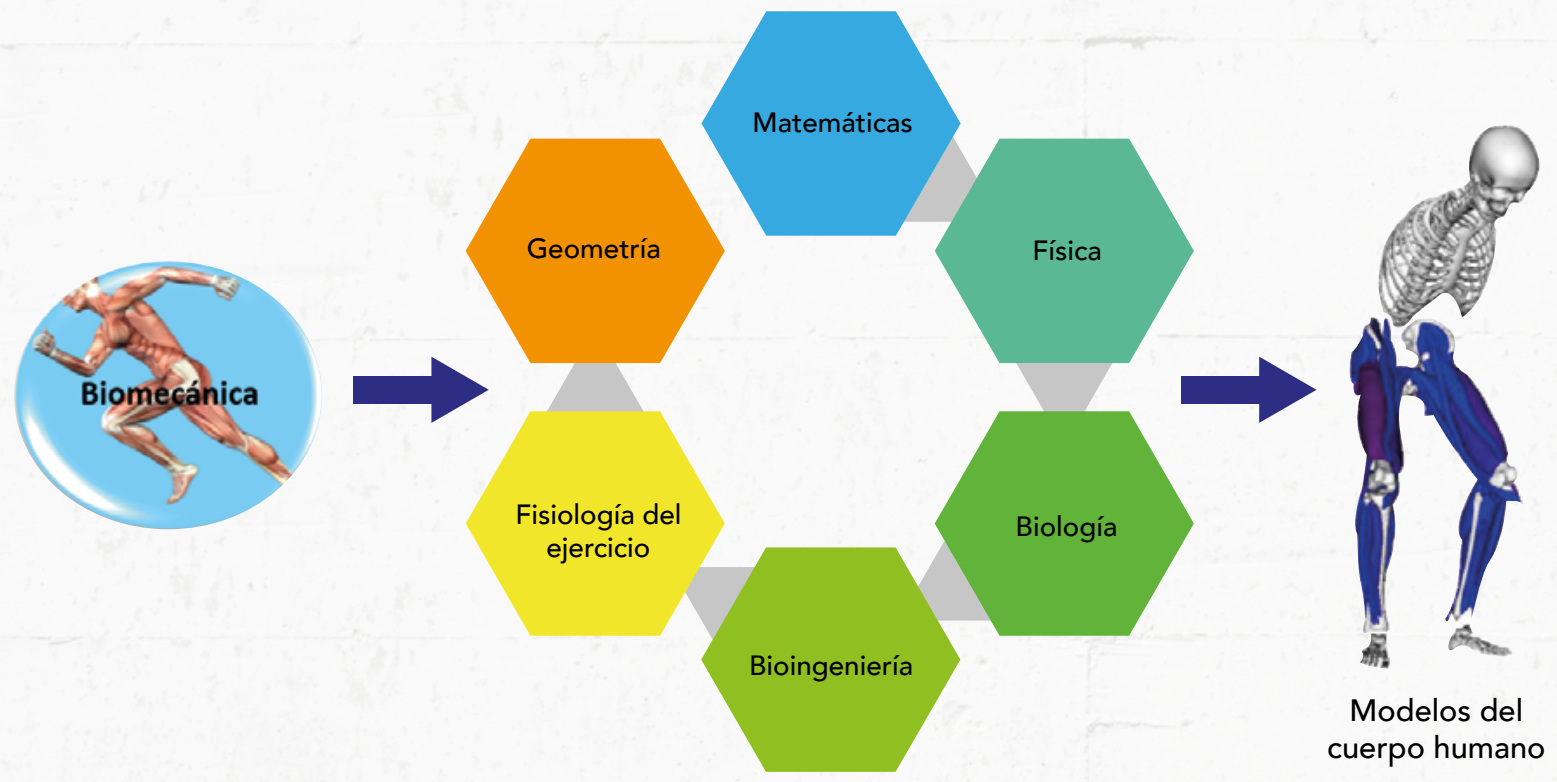

Fuente. Elaboración propia con información tomada de Perdomo-Ogando, Sánchez, Gerónimo y Capote-Domínguez, 2018. 
En este sentido, los desarrollos de la biomecánica, en cuanto línea de estudio e investigación, han servido como base para el diseño de dispositivos, lineamientos y ayudas funcionales que propenden a mejorar el rendimiento deportivo, evaluar condiciones de trabajo, contribuir a la rehabilitación física y la ejecución de cirugías ortopédicas, así como al diseño de prótesis y órtesis (Hirsh, Morisano y Peterson, 2008; Sanabria y Patiño, 2013), puesto que ha influenciado la investigación de matemáticos, físicos, biólogos, médicos e ingenieros, lo cual exalta las características transdisciplinares de esta área y su gran contribución al desarrollo científico y tecnológico (Perdomo-Ogando et al., 2018).

No obstante, y a pesar del avance de estas aplicaciones en los escenarios académicos, la comprensión de la biomecánica es limitada puesto que se han identificado algunas dificultades por parte de los estudiantes, en las didácticas empleadas por el profesor y en el proceso de enseñanza, tal como se ilustra en la tabla 1.

Tabla 1. Dificultades en el proceso de enseñanza-aprendizaje de la biomecánica

\section{Dificultades del proceso de enseñanza}

- Escasez de textos de didáctica de la biomecánica.

- Se ha incursionado en el trabajo con tecnologías de la información y la comunicación (TIC) como elemento de laboratorio, pero la fundamentación pedagógica aún resulta insuficiente.

- Los software especializados son creados para el análisis biomecánico, más no para el aprendizaje del área.

- Algunas instituciones trabajan la enseñanza de la biomecánica sin espacios de práctica.

rán

\begin{tabular}{|l|}
\hline \multicolumn{1}{|c|}{ Dificultades del profesor } \\
\hline - Se evidencian sucintos cambios en los \\
contenidos y en la forma de exponerlos a los \\
estudiantes; esta, en muchos de los casos, se \\
basa en clases expositivas sin interacción con \\
los estudiantes. \\
- \\
- \\
me todos los docentes conocen las TIC que \\
Se evidencia resistencia frente al desarrollo \\
de proyectos de investigación formativa en \\
el área. \\
No todos los docentes están dispuestos \\
a realizar los esfuerzos para mejorar sus \\
estrategias de enseñanza. \\
Algunos docentes muestran indiferencia \\
frente al desarrollo de estrategias \\
pedagógicas para la enseñanza.
\end{tabular}

\section{Dificultades del estudiante}

- Escaso dominio de los preconceptos básicos -matemáticas, física y biología, entre otros-.

- Limitada capacidad para analizar situaciones propias del curso y ejercicios con sucintos cambios respecto a los ejemplos dados en clase.

- Dificultad en asociar contenidos de otras disciplinas con la biomecánica.

- Escasa capacidad de análisis temporoespacial.

- Dificultad para analizar datos recopilados en tablas y gráficos.

- Escasa capacidad para asociar el concepto con lo observado en la práctica.

- Algunos estudiantes manifiestan escasa preocupación y motivación por la asignatura.

Fuente. Cruz-Rodríguez, Flórez-González, J. P.; Gómez, G.; Marysol, 2015; González-Carbonell et al., 2014. 
Lo descrito en la tabla 1 también se evidencia en las escuelas de ingeniería biomédica, en las que se encuentra una desarticulación en los procesos de enseñanza-aprendizaje de la biomecánica, lo cual constituye una preocupación constante para algunos docentes que quieren proponer innovaciones en la enseñanza en esta área (González-Carbonell, Nápoles-Padrón, Calderín-Pérez, Cisneros-Hidalgo y Landín-Sorí, 2014). Con esto se pone de manifiesto la necesidad de que los estudiantes, a través del desarrollo de unas prácticas académicas acordes con los cambios tecnológicos, sociales y culturales, integren sus saberes a fin de comprender fenómenos biomecánicos de forma transdisciplinar (Nápoles-Padrón, González-Carbonell, Chacon y Hernández-de la Torre, 2015). Así, los discentes podrían desarrollar un pensamiento científico e incrementar su creatividad y su capacidad innovadora, en el propósito de trascender las barreras de la disciplina e interpretar su entorno como un todo integrado en el que la realidad es variante, compleja y caótica (Martín, 2019).

En este sentido, las características descritas muestran la necesidad de revisar la forma como el profesor propone y desarrolla los cursos de biomecánica dirigidos a ingenieros biomédicos, más aún cuando el avance de la ciencia y la tecnología funge como eje de desarrollo humano con el fin de que las personas estén en capacidad de comprender e inferir los diversos cambios culturales, sociales, económicos y políticos, entre otros (Balthazard, Currat y Degache, 2015). Lo anterior evidencia la necesidad de motivar en las aulas una cultura científica y tecnológica que lleve al estudiante a comprender la complejidad y la globalidad de la realidad contemporánea con el objetivo de orientarlo para que, de manera autónoma, pueda desarrollar habilidades que le permitan interactuar y desenvolverse en su cotidianidad académica y familiar, así como en su entorno laboral y productivo (Tamayo-Alzate, 2014).

En este sentido, uno de los caminos para abordar el problema identificado se relaciona con el hecho de aplicar las herramientas tecnológicas como mediación pedagógica, con las cuales el docente puede propiciar escenarios que brinden la posibilidad de modificar y, muy seguramente, mejorar las formas de enfrentar el proceso de enseñanzaaprendizaje de las biociencias (Ariza-Nadjar, 2013). Lo anterior en razón a que estos recursos de apoyo académico permiten la interacción de los fenómenos físicos con entornos digitales alimentados que, entre otros aspectos, conducen a reducir el tiempo de registro y el procesamiento de la información obtenida en los experimentos que realizan los discentes en el curso de biomecánica. De esta manera, enfocan su atención en el análisis e interpretación de la información resultado de la experiencia. Esta información la pueden observar a través de la presentación de imágenes, gráficas, tablas, animaciones, etc., para luego asociar con mayor versatilidad y asertividad situaciones en dos y tres dimensiones, así como realizar mediciones más precisas, de modo que optimicen el tiempo y establezcan relaciones de las magnitudes físicas involucradas, con todo lo cual podrán realizar inferencias de los fenómenos estudiados (Villada y Muñoz, 2014).

Lo anterior evidencia la pertinencia de la propuesta didáctica que a continuación se describe, en la medida en que es una estrategia dirigida a aportar al mejoramiento de la comprensión, del análisis en el curso de biomecánica y de sus mecanismos. 


\section{Fases de implementación del uso de software como estrategia didáctica}

Dados los vertiginosos cambios científicos y tecnológicos a los que se ven enfrentados los actores de la educación, estudiantes, docentes, instituciones y familia- (García, Ruiz y García, 2016), es evidente que la implementación de las tecnologías de la información y la comunicación (TIC), así como de las tecnologías para el aprendizaje y el conocimiento (TAC), por sí mismas no resultan efectivas a la hora de proponerlas como un elemento didáctico sin dotarlas de un trasfondo asociado con la planificación y el acompañamiento del docente, a fin de buscar que este tipo de propuestas puedan tener alguna repercusión positiva en el aprendizaje de los estudiantes (Martín, 2019). En este sentido, la didáctica ejecutada en esta experiencia se desarrolló en tres fases, tal como se ilustra en la figura 2 y se describe a continuación.

Figura 2. Fases de desarrollo de la didáctica propuesta

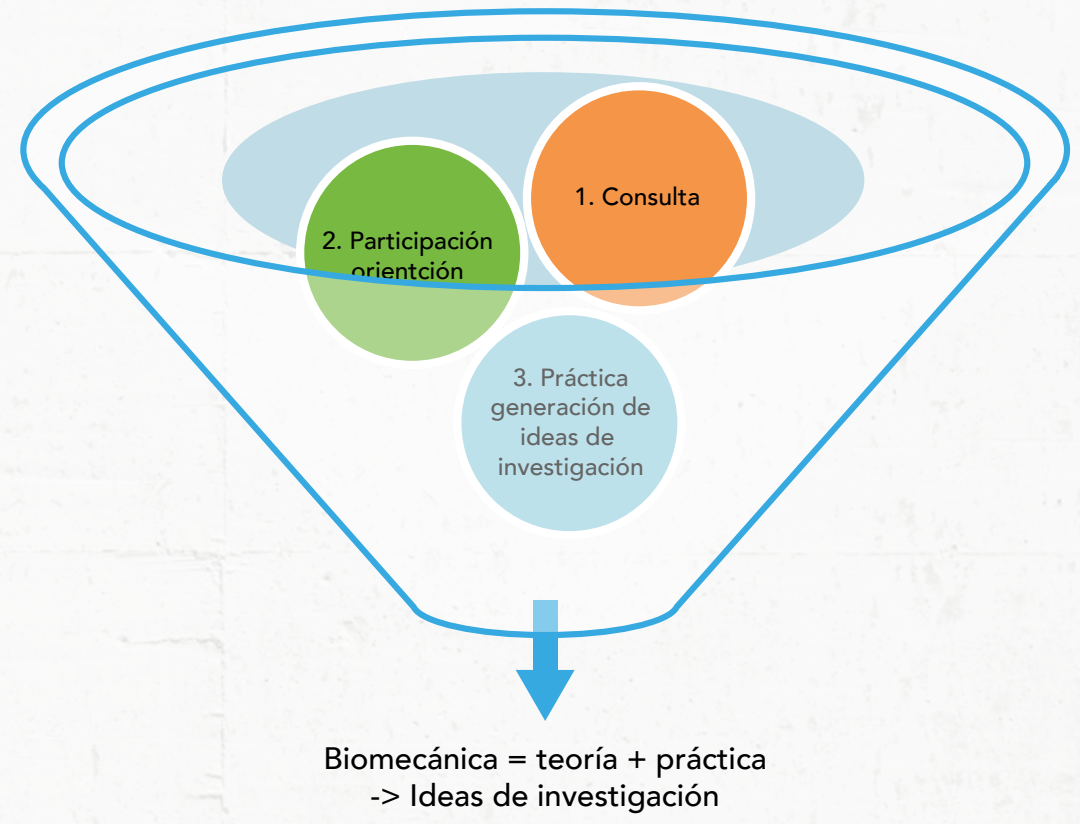

Fuente. Elaboración propia. 


\subsection{Consulta}

En esta fase, que se realiza previo al encuentro de clase, el estudiante desarrolla una revisión sistemática del material de apoyo que el profesor le proporciona (González-Carbonell et al., 2014), así como del material que él mismo busca en las bases de datos académicas y la información resultado de investigación disponible en la red y relacionada con artículos, textos, capítulos de libro o informes de organizaciones médicas, entre otros.

\subsection{Fase de participación-orientación}

Luego de la consulta, el discente se presenta al aula de clases preparado para contestar las preguntas problematizadoras propuestas por el profesor y que en la sesión presencial expondrá ante el grupo de compañeros. En este espacio se comparten ideas, se amplía información y se satisfacen las dudas. Como cierre de esta primera actividad se observan videos resultados de investigaciones y aplicaciones desarrolladas por entidades nacionales e internacionales, pioneras en actividad biomecánica y relacionadas con las temáticas que se trabajan procesualmente en el curso (Cruz-Rodríguez et al., 2015).

\subsection{Fase práctica-generación de ideas de investigación}

Para el desarrollo de esta fase tanto el profesor de teoría como el de práctica trabajan en consonancia en el propósito de desarrollar guías de laboratorio basadas en la revisión teórica y el uso del software de apoyo para la enseñanza aprendizaje de la biomecánica, en este caso particular en Smart Track. Estas guías posibilitan que el estudiante reconozca los entornos digitales desarrollados y empleados en el estudio de fenómenos biomecánicos (Poveda-Polo, 2011); identifique, con base en su experiencia, los conceptos biomecánicos trabajados en la teoría; y desarrolle la capacidad crítica que le permita analizar, desde lo observado, las características del cuerpo humano. Todo esto valiéndose de elementos de análisis tales como figuras tridimensionales, tablas que pueden representar relaciones de magnitudes físicas independientes e integradas, gráficos y datos, lo cual logra a partir de la aplicación de diversas rutinas por las que logra integrar conocimientos de anatomía, fisiología, antropometría y física, entre otros. A modo de ejemplo, a continuación se describe una de estas rutinas.

a. Plan para la toma de datos. Cada grupo de trabajo, compuesto por cuatro estudiantes, elabora un modelo en papel de los puntos anatómicos que representan el estudio de una temática de la biomecánica en particular, como, por ejemplo, el estudio cinético y cinemático de las articulaciones corporales en miembro inferior, tal como se aprecia en la figura 3. 
Figura 3. Mapa de reconocimiento anatómico del miembro inferior

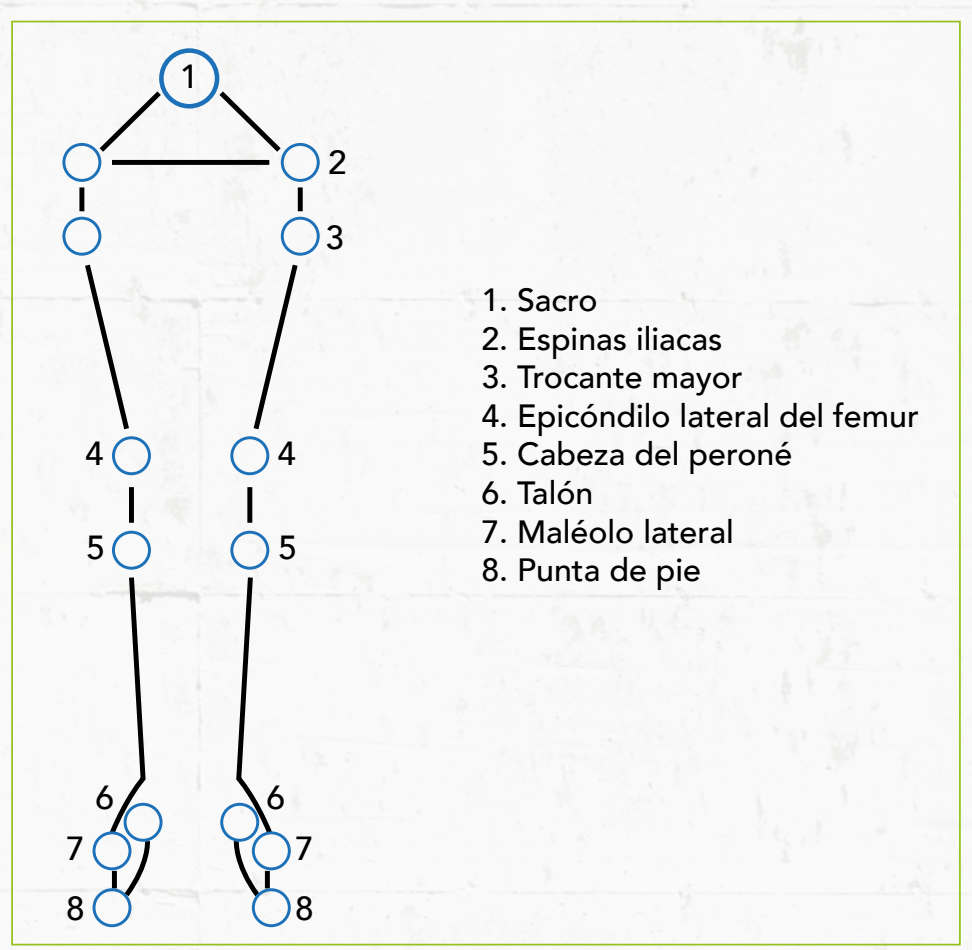

Fuente. Elaboración propia.

b. Uso de la interfaz análoga - cuerpo humanodigital —software-. Para esta actividad los estudiantes hacen uso de marcadores o sensores ${ }^{3}$ que deben colocar cuidadosamente sobre el paciente o persona objeto de estudio, en los puntos anatómicos que caracterizan el tema de interés a revisar — como es el caso del miembro inferior-, en el orden que muestra la figura 4 y en conformidad con el plan de toma de datos expuesto en el ítem c. Con el uso de esta tecnología el estudiante puede lograr que algunas regiones corporales sobresalgan para que la luz se refleje con ayuda de las cámaras, que son las encargadas de tomar la secuencia fotográfica de los movimientos. De esta manera, el discente puede reconstruir la secuencia de movimientos y percibir detalles biomecánicos que a simple vista pasarían desapercibidos, así como comprender los movimientos característicos del miembro inferior desde 
los grados de libertad de las articulares, las capacidades musculares y óseas, y determinar situaciones regulares y posibles alteraciones patológicas. También puede identificar los conceptos de estabilidad y equilibrio de acuerdo con el análisis de centro de gravedad y de masa.

Figura 4. Disposición de marcadores en el sujeto objeto de estudio

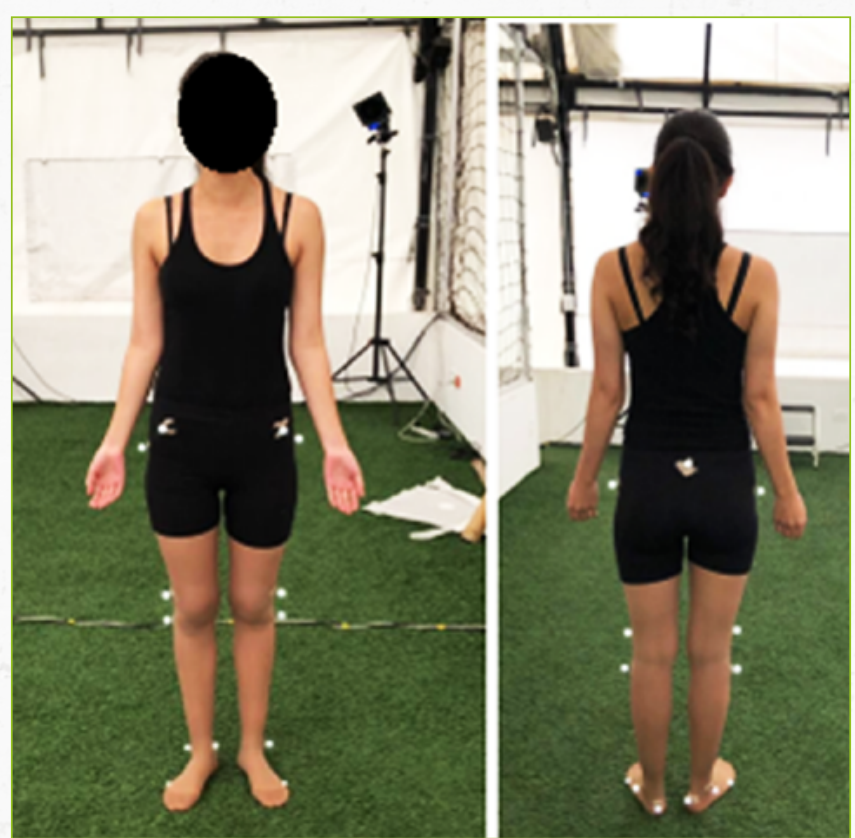

Fuente. Elaboración propia.

c. Digitalización del movimiento. Una vez el estudiante ha realizado el proceso de toma de datos el software realiza la captura del movimiento — smart capture-, el cual se digitaliza a través de los marcadores en el espacio y en el tiempo, con lo cual el estudiante logra observar la imagen que se capturó en tres dimensiones, además de la posición, el desplazamiento y la aceleración de cada uno de los marcadores. De esta manera puede establecer relaciones y conceptos de matemática y física aplicados al cuerpo humano, tal como se puede apreciar en la figura 5. 
Figura 5. Movimiento recreado en 3D de miembro inferior

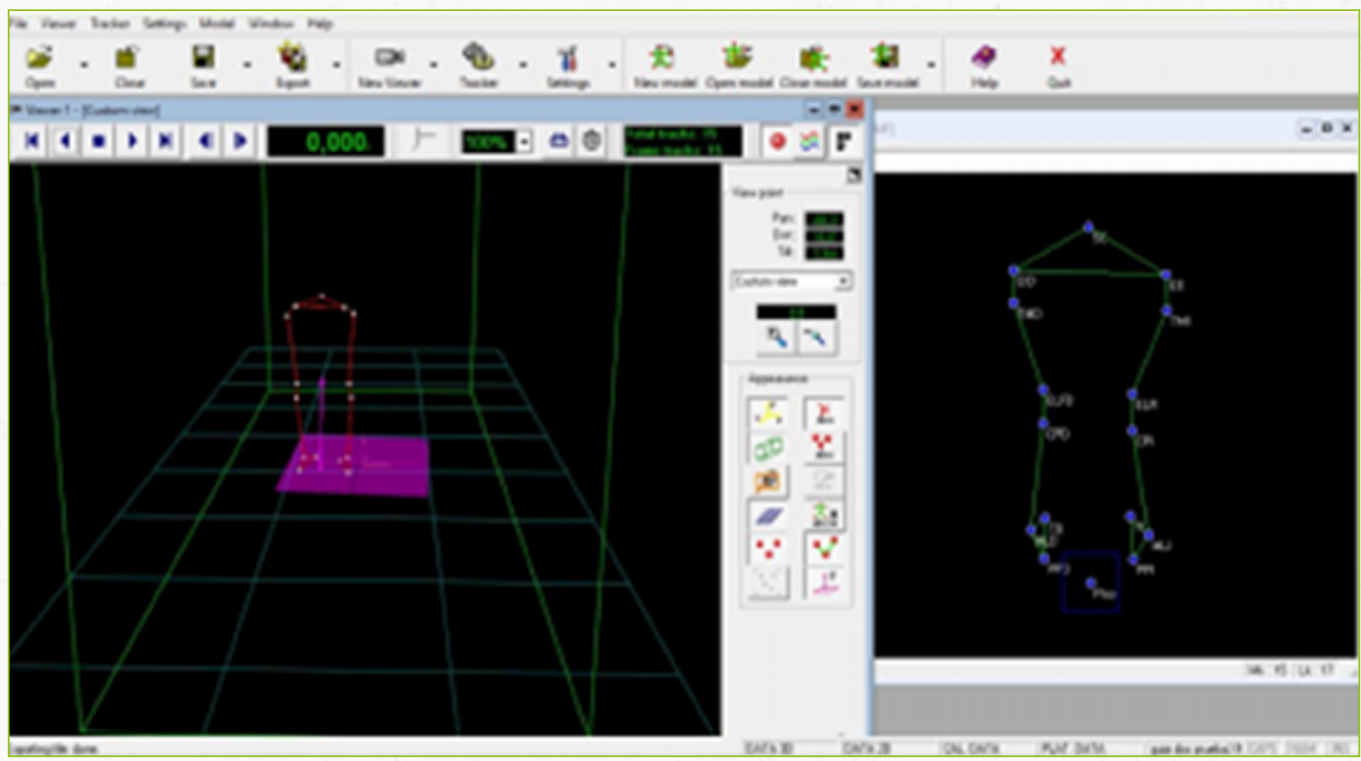

Fuente. Elaboración propia.

d. Observación, descripción y análisis de datos. Luego de la obtención del modelo tridimensional el estudiante elabora las gráficas solicitadas en la guía de laboratorio y aquellas que resulten de su interés a fin de que esté en capacidad de responder las preguntas problematizadoras - como es el caso de la posición versus el tiempo-, referentes a los indicadores que con colores diferencian los puntos anatómicos que se establecieron en la fase de diseño. Por otra parte, los datos obtenidos y presentados en tablas se relacionan con las gráficas y corresponden a los movimientos realizados en los diferentes ejes - x,y y $z-$, respectivamente. Así, por ejemplo, como se muestra en la figura 6, la roja es la espina iliaca derecha, la verde el epicóndilo lateral izquierdo y el azul el talón izquierdo; de esta forma, el estudiante visualiza los puntos antropométricos, los relaciona con parámetros físicos y los analiza desde el punto de vista biomecánico, lo cual le permite observar la situación de forma integral (Rojo, 2015). 
Figura 6. Relaciones de velocidad, aceleración y posición acordes con los movimientos estudiados

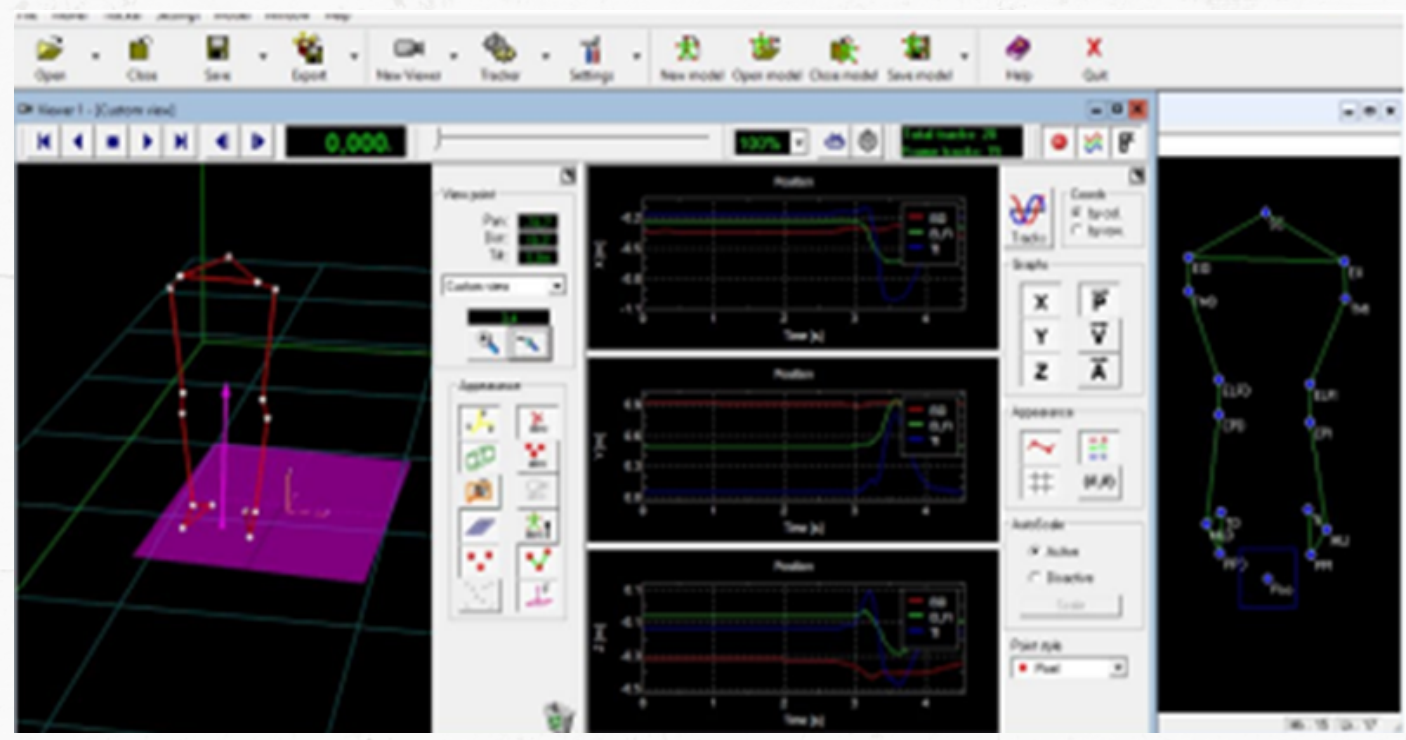

Fuente. Elaboración propia.

\section{Conclusiones}

Con la experiencia descrita los estudiantes participantes fueron expuestos a la contrastación de la teoría con la práctica, al hacer uso de una ruta metodológica que los enfrentó al reto a ser hacedores de su propio conocimiento a través de la indagación. Esto en razón a que fueron responsables de preparar la temática a tratar por medio de diferentes recursos - lecturas, videos, etc.-que sirvieron de apoyo para que ellos realizaran sus propias comprensiones, inferencias y preguntas, de manera que, al implementar la tecnología, observaron con mayor precisión los fenómenos y complementaron sus análisis iniciales. Con esto se logró minimizar la reprobación académica en un $35 \%$ con relación al histórico de los cursos desde el 2010 al 2017.

La didáctica sugerida condujo a los estudiantes a analizar de manera asociativa la construcción del movimiento humano regular —normal- y tomar en cuenta, a su vez, los mecanismos osteo-artro-neuromusculares, para lo cual 
el discente debió asociar preconceptos relacionados con anatomía descriptiva, fisiología, histología y biofísica, lo que le imprime a esta ciencia un carácter transdisciplinar, pues la permean elementos de otras ciencias y de otras áreas, en consonancia con los factores sociales, culturales y económicos de la población que se traducen en la construcción de la mecánica corporal asociada a la función.

El análisis y el uso de recursos matemáticos que el estudiante debió realizar contribuyó a la interpretación de gráficas en sistemas de coordenadas, de acuerdo con el tipo de función que representa el fenómeno biomecánico estudiado, además de observar y analizar las dependencias y el comportamiento de las magnitudes biofísicas y los objetos de medición con los que el estudiante interactúa durante las prácticas.

De igual modo, la manipulación del software por parte del estudiante conduce a la digitalización de los movimientos corporales analizados, lo que le permite calcular una gran cantidad de variables cinemáticas tales como el centro de gravedad, los ángulos absolutos y relativos, el desplazamiento, las velocidades y aceleraciones, todo lo cual, a su vez, hace más preciso el análisis de movimiento.

La interacción con el programa especializado también posibilitó que el estudiante estuviera en capacidad de calcular diferentes variables dinámicas, como, por ejemplo, energías del sistema y el momentum, las fuerzas de reacción de las articulaciones y el torque.
En cuanto a la construcción de los modelos tridimensionales, aportan a la comprensión del área puesto que permiten al estudiante observar el movimiento realizado en el espacio - $x ; y ; z-$, de modo que lo asocia a la realidad corporal y temporo-espacial.

Respecto a la formación investigativa, dada la experiencia en biomecánica, el uso de software y el desarrollo de la capacidad analítica, los estudiantes de ingeniería biomédica pueden diseñar o proponer proyectos orientados a desarrollar dispositivos de asistencia para el diagnóstico, la rehabilitación y la evaluación con base en la biomecánica, lo cual les permita resolver problemáticas con respecto al ambiente laboral, la preparación deportiva y de discapacidad en el país.

Los procesos de innovación enseñanza-aprendizaje de la biomecánica en escuelas de ingeniería favorece en los estudiantes el desarrollo de sus capacidades de observación, análisis, razonamiento, comunicación y abstracción, de modo que los conduce a que construyan su aprendizaje con gran autonomía.

El hecho de que estructuren microcurrículos que permitan encontrar una cohesión entre la práctica y la teoría incentiva que el estudiante encuentre una articulación de su plan de estudios y se forje la cultura científica en el futuro ingeniero, lo que lo conduce a desarrollar su personalidad individual y social. 
Así mismo, la implementación de software especializado de análisis de movimientos como mediación pedagógica propicia escenarios para mejorar la atención, el interés, el análisis y la inferencia de fenómenos biomecánicos que aproximan al estudiante a la realidad natural, a la interacción con la tecnología de diagnóstico, al contexto profesional y a pensar en sociedad, así como a contribuir a las problemáticas que pueden encontrarse en los ejercicios de investigación.

La contribución del software en el análisis de movimiento en situaciones biomecánicas patológicas es una oportunidad para que el estudiante comprenda las condiciones de adaptación del cuerpo humano, lo cual conduce al desarrollo de habilidades especiales que son de gran interés para proponer diseños de ayudas funcionales y la creación de dispositivos biomédicos. Esto puede mejorar la calidad de vida de las personas e incrementar los desarrollos de ingeniería biomédica, de modo que genere la proyección de 15 propuestas de trabajo de grado relacionadas con ayudas funcionales, prótesis transfemoral, dispositivos de rehabilitación, análisis de puestos de trabajo y protección industrial.

\section{Referencias}

Aguilar, M.; Gutiérrez, M. A. (2000). Biomecánica, la física y la fisiología. Madrid: Editorial CSIC-CSIC Press.

Ariza-Nadjar, A. (2013). Elaboración de un libro texto y material de laboratorio para la optativa introducción a la ingeniería biomédica (Tesis de grado). Universidad Pontificia Bolivariana. Bucaramanga, Colombia. Recuperado de https://bit. ly/2VakbKc
Balthazard, P.; Currat, D.; Degache, F. (2015). Fundamentos de biomecánica. EMC-Kinesiterapia-Medicina Física, 36(4), 1-8. DOI: https://doi.org/10.1016/S1293-2965(15)74142-3

Bergós, L.; Cabrera, C. (2014). Aprendizaje basado en proyectos para estudiantes de biociencias de la Facultad de Ciencias de la Universidad de la República, Uruguay. Ponencia presentada en la XI Jornadas Nacionales y VI Congreso Internacional de Enseñanza de la Biología. General Roca, Río Negro, Argentina, 11-14 de octubre.

Cruz-Rodríguez, J. L.; Flórez-González, J. P.; Gómez, G.; Marysol, L. (2015). Metodologías que se utilizan en el espacio académico análisis de movimiento y biomecánica en la licenciatura en educación física recreación y deporte de Uniminuto (Tesis doctoral). Corporación Universitaria Minuto de Dios. Bogotá, Colombia. Recuperado de https://bit.ly/2K6uz4I

Donskoi, D.; Zatsiorski, V. (1988). Biomecánica de los ejercicios físicos. Moscú: Editorial Raduga.

Dufour, M.; Pillu, M. (Eds.). (2018). Biomecánica funcional. Miembros, cabeza, tronco. Polonia: Elsevier.

Frutos, J. B.; Palao, J. M. (2012). El uso de la videografía y software de análisis del movimiento para el estudio de la técnica deportiva. Lecturas: Educación Física y Deportes, 17(169), $1-14$.

García, L.; Ruiz, M.; García, M. (2016). Claves para la educación: actores, agentes y escenarios en la sociedad actual (Vol. 3). Madrid: Narcea Ediciones.

González-Carbonell, G.; Nápoles-Padrón, E., Calderín-Pérez, B., Cisneros-Hidalgo, Y.; Landín-Sorí, M. (2014). Carácter interdisciplinario de la modelación computacional en la solución de problemas de salud. Humanidades Médicas, 14(3), 646-658. 
Hirsh, J. B.; Morisano, D.; Peterson, J. B. (2008). Delay discounting: interactions between personality and cognitive ability. Journal of Research in Personality, 42(6), 1646-1650. DOI: https:// doi.org/10.1016/j.jrp.2008.07.005

Martín, J. (2019). Aprendizaje transdisciplinar de las ciencias matemáticas mediado por realidad aumentada en programas de ingeniería (Tesis doctoral). Universidad Santo Tomás. Bogotá, Colombia.

Nápoles-Padrón, E. N.; González-Carbonell, R. G.; Chacon, M.; Hernández-de la Torre, J. (2015) La investigación interdisciplinaria: un ejemplo en el grupo de bioingeniería y biomecánica. Ponencia presentada en la VI conferencia Internacional de Ciencia y Tecnología por un desarrollo sostenible. Universidad de Camagüey Ignacio Agramonte Loynaz - Camagüey - Cuba, 3 a 5 de junio.

Perdomo-Ogando, J. M.; Sánchez, P.; Gerónimo, A.; CapoteDomínguez, T. E. (2018). Premisas para la investigación biomecánica en la cultura física. Revista Cubana de Educación Superior, 37(2), 104-114.

Poveda-Polo, A. (2011). Los objetos de aprendizaje: aprender y enseñar de forma interactiva en biociencias. ACIMED, 22(2), 155-166.

Rojo, D. C. V. (2015). La interdisciplinariedad: necesaria en la creación de intereses congnoscitivos por el estudio de la física. III Conferencia Científica Internacional de la UNISS. Universidad de Sancti Spíritus. Sancti Spíritus, Cuba, 19-21 de noviembre. Recuperado de https://bit.ly/3erAqQ7

Sanabria, N. S.; Patiño, A. M. O. (2013). Biomecánica del hombro y bases fisiológicas de los ejercicios de Codman. Ces medicina, 27(2), 205-218.
Tamayo-Alzate, Ó. E. (2014). Pensamiento crítico dominio-específico en la didáctica de las ciencias. Tecné Episteme y Didaxis TED, (36), 25-46. DOI: https://doi.org/10.17227/01203916.4686

Villada, F.; Muñoz, J. (2014). Desarrollo de un software de análisis biomecánico a través de datos de captura de movimiento usando el sensor KINECT para rehabilitación asistida con video juegos. Entre Ciencia e Ingeniería, 8(16), 72-77. 